\title{
Analysis of crack mechanism in FGH 98 superalloy during the laser solid forming process
}

\author{
Weisheng Ying ${ }^{1,2}$, Shukai Fan ${ }^{1,2}$, Fuzhu Han ${ }^{1,2,{ }^{*}}$ and Junhua Wang ${ }^{1,2}$ \\ ${ }^{1}$ Department of Mechanical Engineering, Tsinghua University, Beijing 100084, China \\ ${ }^{2}$ Beijing Key Lab of Precision/Ultra-precision Manufacturing Equipments and Control, Tsinghua University, Beijing 100084, China
}

\begin{abstract}
In this study, FGH 98 superalloy parts were fabricated by laser solid forming (LSF) and the microstructure and defects of the LSF part were revealed using optical microscope and scanning electron microscope. It was found that the main defect in the LSF part was cracks. In order to analyze the phase transition process and crack mechanism, the temperature and stress fields were simulated. Based on the simulated result, Ni-Al phase diagram and morphologies of cracks, it can be concluded that the micro segregation caused by the rapid heating and cooling rates was the main reason for the crack defect.

Key words. FGH 98 superalloy; Microstructure; Defects; Crack mechanism; Laser solid forming;
\end{abstract}

\section{Introduction}

With the development of aeroengines, the increase of the working temperature demands more superior hightemperature mechanical properties for the material of aeroengine components. FGH 98, a third-generation powder metallurgy nickel-based superalloy, has been widely used for manufacturing aeronautical components due to its good balance between creep resistance, damage tolerance, and tensile properties at elevated temperatures [1]. However, due to the high concentration of refractory elements and the inherent interaction capabilities among the complex precipitation strengthening phases in the FGH 98 superalloy, it is difficult to fabricate components with complex shapes using traditional processing methods $[2,3]$. In recent years, additive manufacturing (AM) technologies have been developed to produce complex-shaped parts due to its advantages in low production cost, low waste ratio of material and short production cycle. Laser solid forming (LSF), also known as laser metal deposition (LMD) or laser engineered net shaping (LENS), is a typical additive manufacturing technology that combines the advantages of rapid prototyping and laser cladding. Compared to the traditional processing method, LSF can achieve near net shape forming of components without die. Given the notable improvement in production flexibility, LSF is a promising method for manufacturing compact metallic parts with high performance [4].

However, during the rapid heating and cooling process of LSF, there is an inhomogeneous thermal field and steep thermal gradient in the fabricated parts, which leads to a nonequilibrium solidification process and severe stress concentration. Therefore, because of the rapid heating and cooling characteristics, severe defects have a big chance to occur in the LSF part [5]. For FGH 98 superalloy, there are few researches concerning about using LSF to fabricate FGH 98 superalloy parts. The kinds of defects, which may occur in the LSF FGH 98 part, are still unclear. Thus, it is meaningful to have a new attempt to fabricate FGH 98 parts by LSF.

In this study, FGH 98 cuboid samples were fabricated by LSF and defects in the sample were revealed. The temperature field and stress field of the LSF process were simulated by ANSYS software. Based on the characteristics of simulation results and phase diagram of FGH98 superalloy, the microstructure evolution and crack mechanism in the LSF FGH98 superalloy part were explained.

\section{Experimental setup}

All the LSF experiments involved in this study were performed on a self-developed LSF equipment, which consisted of a SPI $400 \mathrm{~W}$ continuous wave laser, a computer numerical control (CNC) working table and a coaxial powder feeding system. FGH 98 spherical powders with particle size in the $45-100 \mu \mathrm{m}$ range were laser-deposited on a $150 \mathrm{~mm} \times 150 \mathrm{~mm} \times 8 \mathrm{~mm}$ stainless steel substrate. Table 1 presents the chemical composition of FGH 98 superalloy. The substrate surface was ground using $\mathrm{SiC}$ abrasive paper and cleaned using absolute ethanol in an ultrasonic bath before being laser-deposited. To observe the microstructure of the cross sections, the samples were sectioned using the method of wire electrical discharge machining (WEDM). The metallographic specimens were prepared using standard metallographic techniques, and the etchant used for

hanfuzhu@mail.tsinghua.edu.cn 
revealing the microstructure consisted of $50 \mathrm{~mL} \mathrm{H} \mathrm{H}_{2} \mathrm{O}, 50$ $\mathrm{mL} \mathrm{HCl}$, and $10 \mathrm{~g} \mathrm{CuSO}_{4}$.

Table 1. Chemical composition of FGH 98 superalloy (wt \%)

\begin{tabular}{|c|c|}
\hline Element & FGH 98 \\
\hline $\mathrm{Cr}$ & 0.05 \\
\hline $\mathrm{Co}$ & 12.29 \\
\hline $\mathrm{Mo}$ & 20.68 \\
\hline $\mathrm{W}$ & 4.61 \\
\hline $\mathrm{Al}$ & 2.81 \\
\hline $\mathrm{Ti}$ & 3.32 \\
\hline $\mathrm{Ti}$ & 3.71 \\
\hline $\mathrm{Nb}$ & 1.22 \\
\hline $\mathrm{Ta}$ & 2.47 \\
\hline $\mathrm{B}$ & 0.02 \\
\hline $\mathrm{Zr}$ & 0.05 \\
\hline $\mathrm{Ni}$ & Bal. \\
\hline &
\end{tabular}

\section{Defects in the LSF FGH98 superalloy}

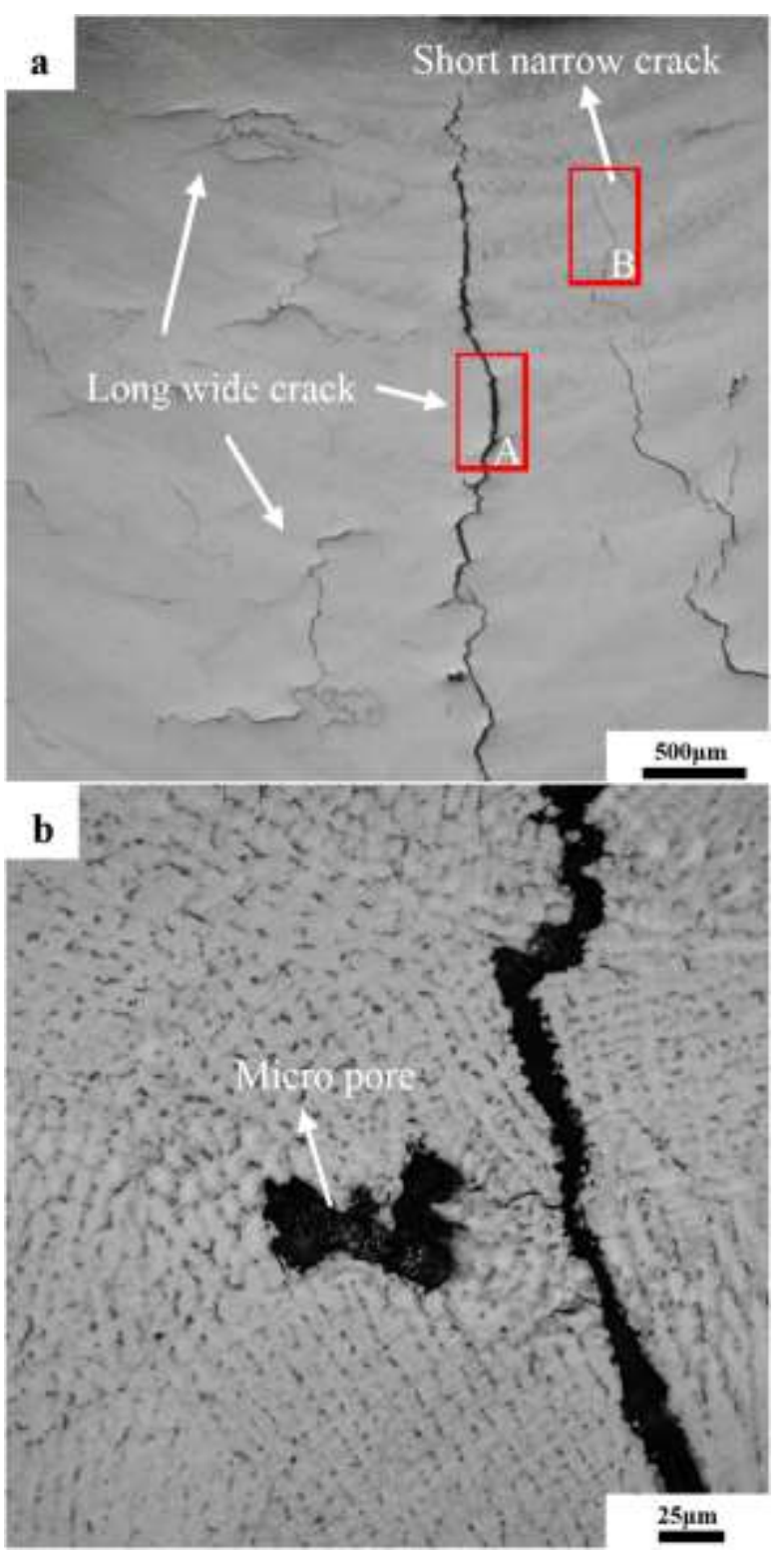

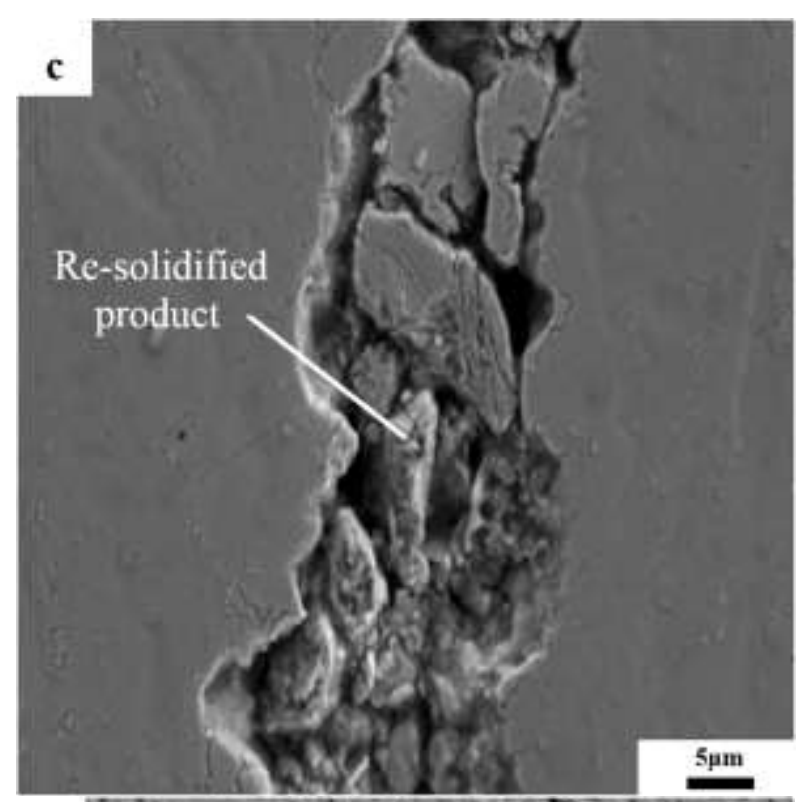

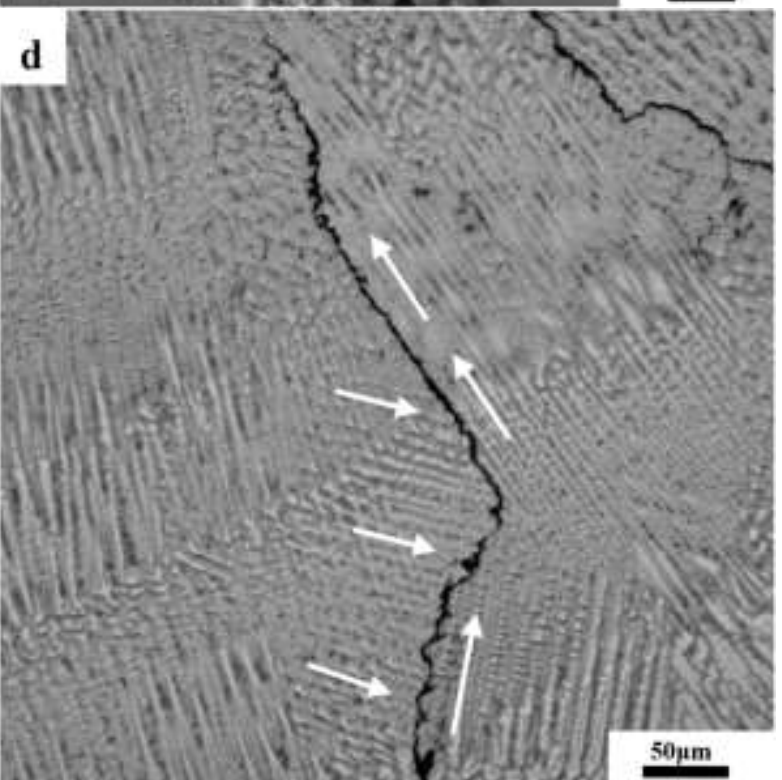

Figure. 1 Microstructure of the LSF FGH 98 superalloy: (a) low magnification OM image, (b) micro pore in the LSF part, (c) highly magnified SEM image of the long wide crack in area A in Figure. 1(a), and (d) highly magnified OM image of the short narrow crack in area B in Figure. 1(a) (the white arrow represents the direction of the grain boundary)

Figure. 1 shows the typical microstructure of the LSF FGH 98 superalloy sample. The microstructure mainly consisted of columnar dendrites which were epitaxially grown from the substrate. Both cracks and micro pores were found on the cross section of the LSF FGH98 part, and the main defect was obviously cracks. Depending on the morphologies of cracks, the cracks can be mainly divided into two types: long wide cracks and short narrow cracks. As shown in Figure. 1(d), it can be found that the short narrow crack initiated in the overlap area between two adjacent scanning passes, where grain boundaries of different directions met, and mainly distributed along grain boundaries. Figure. 1(c) shows the highly magnified SEM images of long cracks. Although the cross direction raster scanning pattern was used in the LSF process, the change of the columnar grain growth 
direction was not so obvious. Some cracks propagated along the crystallographic orientation of the columnar dendrite and traversed several layers to form a long crack. In the long crack, there were a lot of re-solidified products. Comparing to the short crack, the long crack always had some branches and presented a dendritic morphology.

\section{Finite element modeling}
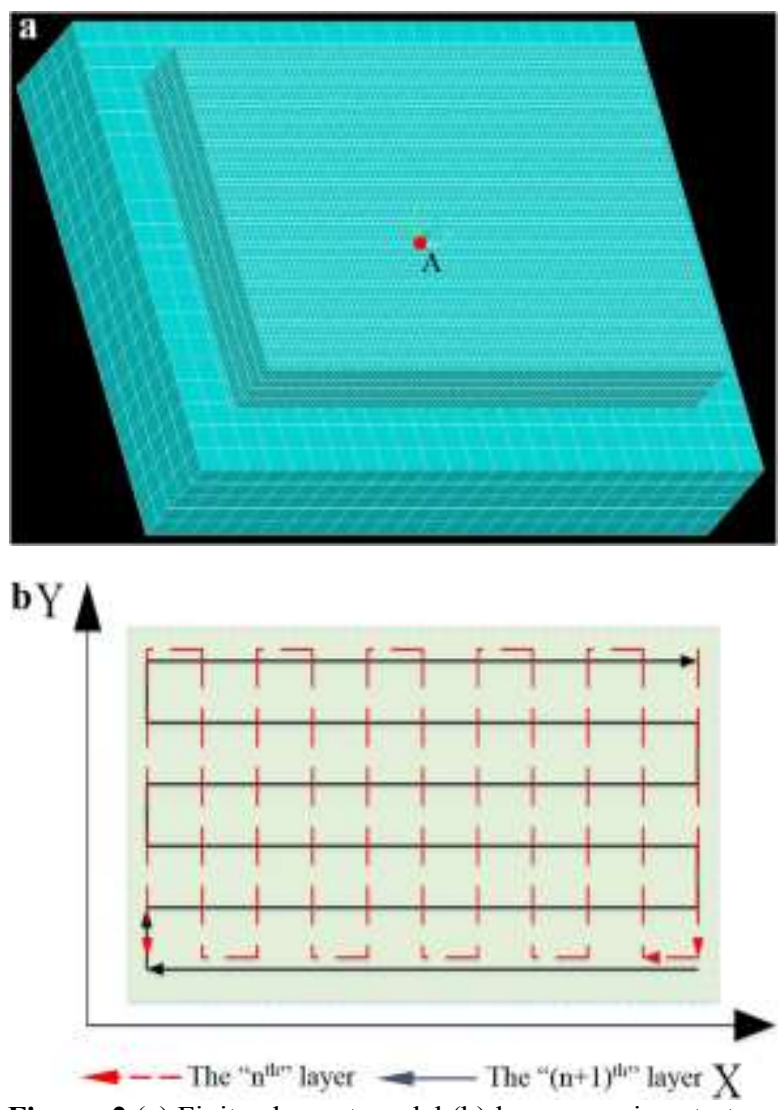

Figure. 2 (a) Finite element model (b) laser scanning strategy

The generation of cracks is decided by the material properties and inner thermal stress. For superalloy, material properties have a close relationship with the temperature. However, it is hard to measure the temperature and stress fields accurately during the LSF process. In order to understand the generation and propagation mechanism of cracks, the temperature and stress fields were simulated by ANSYS software in this study. A $8 \mathrm{~mm} \times 6 \mathrm{~mm} \times 1.2 \mathrm{~mm}$ finite element model is established, as shown in Figure. 2. In order to simulate the transient thermal behaviour and subsequent deposition process, the element birth and death technique of ANSYS was adopted. The variations of temperature and stress in the center point of first layer (marked A in Figure. 2) were picked to analyze the temperature and stress fields during the LSF process. The assumptions about material deposition in the model included:

1) The material deposition speed was assumed to be the same as the laser scanning speed.

2) The substrate size was $10 \mathrm{~mm} \times 8 \mathrm{~mm} \times 2 \mathrm{~mm}$, which was far smaller than the substrate size used in the LSF experiments. Thus, the specific heat of substrate was set higher than the normal value.

3) The material properties of FGH 98 were obtained from Chinese Handbook of Aeronautical Materials.

\section{Temperature and stress variations during the LSF process}
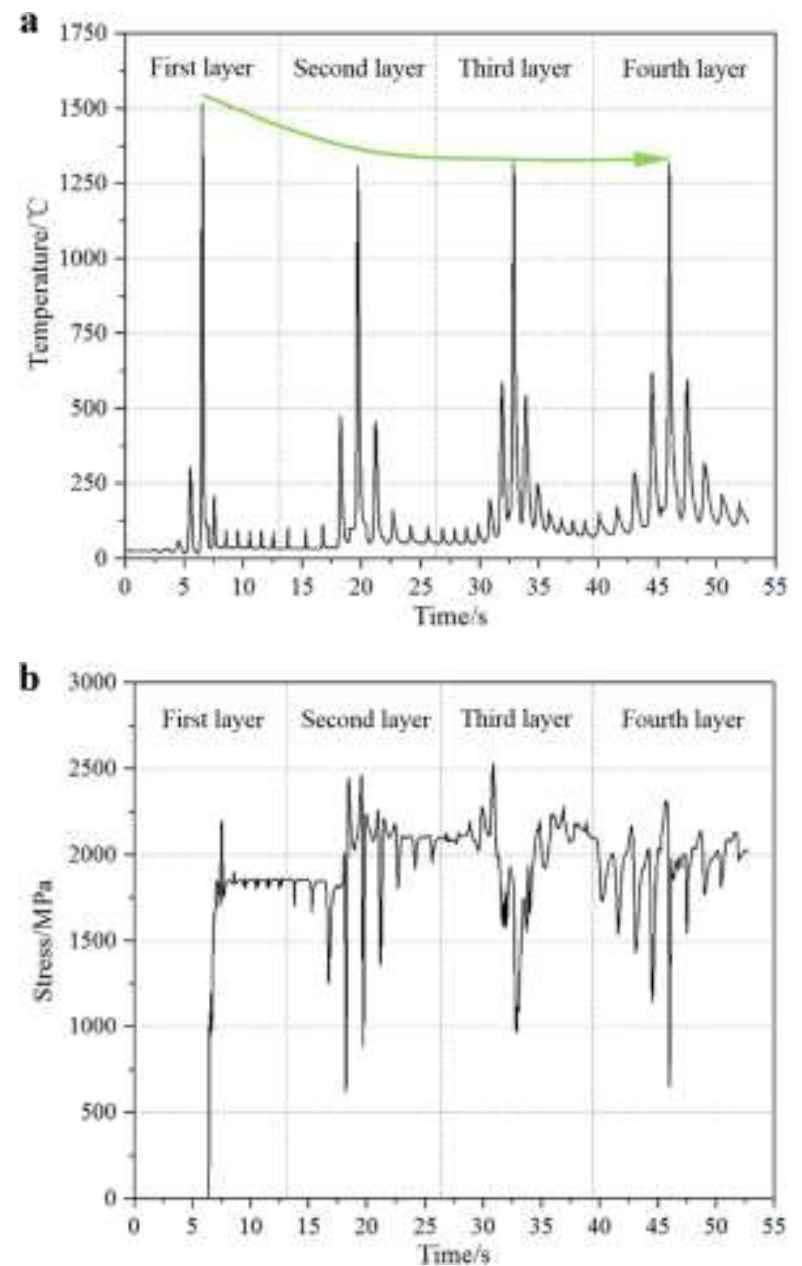

Figure. 3 Temperature (a) and stress (b) variations of point A during the LSF process

Figure. 3 presents the temperature and stress variations of point A during the LSF process. Because of the characteristic of the element birth and death technique, the temperature of point $\mathrm{A}$ was low before the element which point A belonged to was activated. Once activated, it can be found that point A experienced a series of periodic temperature cycles. The temperature variation in different layer was similar. The temperature decreased with the increasing distance between point $\mathrm{A}$ and the laser spot. Every time when the laser spot passed point A, the temperature reached the highest value during the deposition process of each layer. And the peak temperature in each layer showed a general downward trend with the increase of deposition layers. The temperature variation in Figure. 3 has demonstrated that when the laser spot was close to point A, the temperature would exceed the melting point, which meant the material of point A may be remelted for several times during the LSF process. Moreover, from Figure. 3, it can 
be found that the cooling rate was also quite high. The fast heating and cooling rate of point A led to a high temperature gradient. Because of the high temperature gradient, there was a huge temperature difference around the laser spot, which led to non-uniform deformation and severe stress concentration. Thus, because of the periodic temperature cycles, point $\mathrm{A}$ would also experience a series of periodic stress cycles.

\section{Crack mechanism of the LSF FGH98 superalloy}
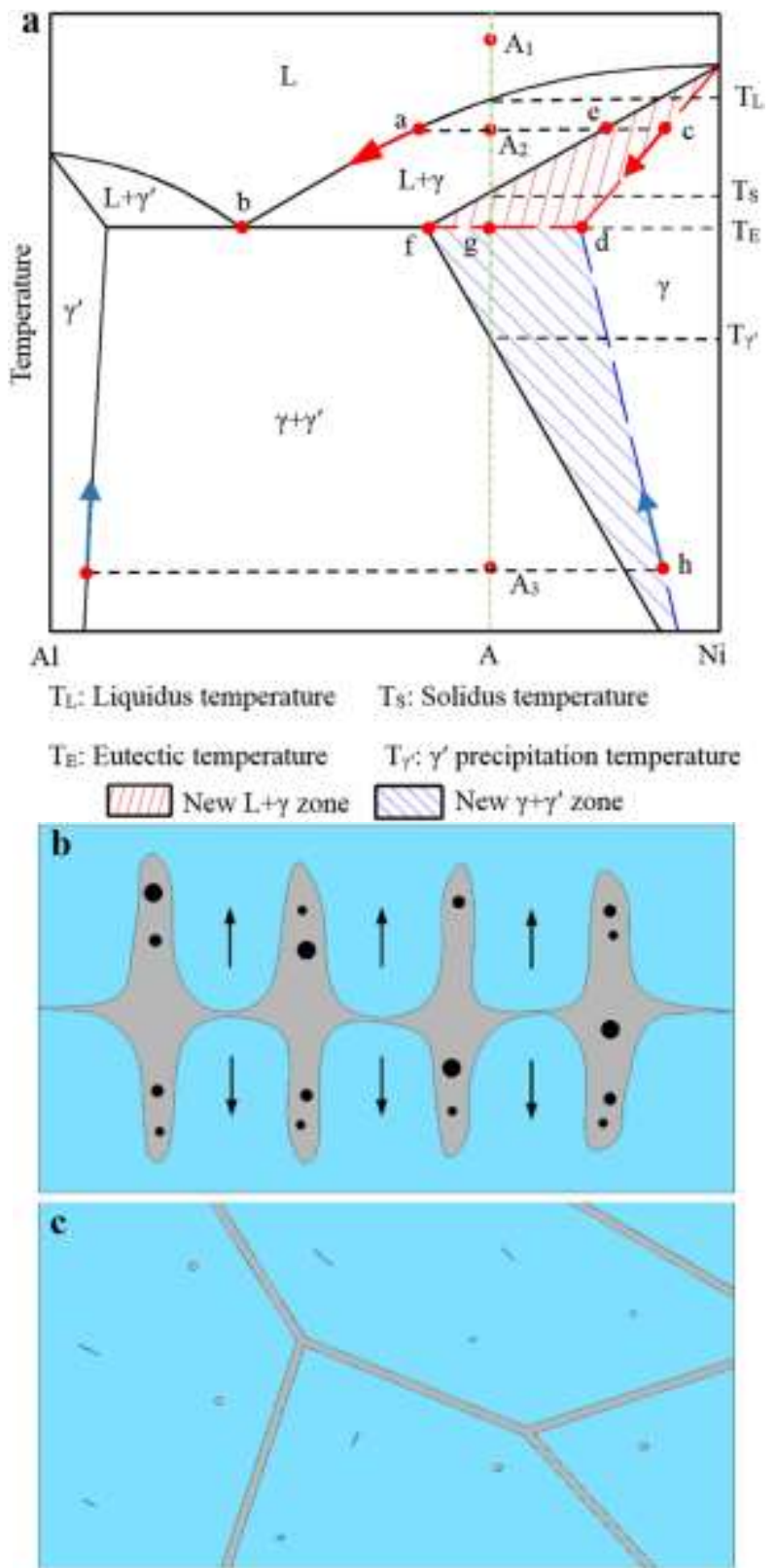

Grain $\ \gamma-\gamma^{2}$ eutectic liquid

$\gamma-\gamma^{\prime}$ eutectic $\longleftarrow$ Thermal stress

Figure. 4 (a) Ni-Al binary phase diagram (b) mixture of $\gamma-\gamma^{\prime}$ eutectic and $\gamma-\gamma^{\prime}$ eutectic liquid in the interdendritic region in the rapid solidification process (c) low-melting point eutectic liquid film along the grain boundary in the reheating process

The crack is mainly caused by the high concentration of $\mathrm{Al}+\mathrm{Ti}$ in superalloy, and the phase transformation behaviors in the Ni-Al or Ni-Ti system are similar [6,7].
Thus, to understand the effect of temperature cycle on material properties, a Ni-Al binary phase diagram was used to analyze the phase transition process. For FGH 98 superalloy, the chemical composition was close to point $\mathrm{A}$ in the phase diagram. When FGH 98 superalloy started to cool down from point A1 or A2, based on the phase diagram it can be found that there would be no $\gamma^{\prime}$ phase under slow cooling rate until the temperature was decreased to the $\gamma^{\prime}$ precipitation temperature. However, according to the simulation result, the cooling rate was very high. During the solidification process, there would be not enough time for Al element to diffuse in the $\gamma$ matrix, which led to a higher $\mathrm{Al}$ content in the liquid. Without considering the uneven diffusion of $\mathrm{Al}$ element in the liquid, the solid component changed following the line cd instead of line ef in the phase diagram while the liquid component still changed with the line ab. When the temperature was decreased to the eutectic temperature, according to the lever rule and new phase diagram, there would be still some liquid in the interdendritic regions, and the liquid component was close to the eutectic point $b$. Thus, during the rapid solidification process, there was a high possibility for the eutectic reaction to occur. As shown in Figure. 4(b), a mixture of $\gamma-\gamma^{\prime}$ eutectic and $\gamma-\gamma^{\prime}$ eutectic liquid might exist in the interdendritic region, which resulted in the decrease in ductility. When the thermal stress overcame the low ductility of the material, the mixture of $\gamma-\gamma^{\prime}$ eutectic and $\gamma-\gamma^{\prime}$ eutectic liquid in the interdendritic region would be torn, causing solidification cracks along the interdendritic regions. According to the analysis above, we thought the long crack may be caused by the rapid solidification process and belong to the solidification crack. The re-solidified product in the long crack was examined by EDS. Based on the EDS results, it can be found that the composition of re-solidified product corresponded with the $\gamma-\gamma^{\prime}$ eutectic, which proved the analysis above.

Table 2. EDS results of the re-solidified product and surrounding material (wt \%)

\begin{tabular}{|c|c|c|}
\hline Element & Re-solidified product & Surrounding material \\
\hline $\mathrm{Cr}$ & 8.31 & 12.32 \\
\hline $\mathrm{Co}$ & 17.69 & 20.53 \\
\hline $\mathrm{Mo}$ & 9.23 & 4.71 \\
\hline $\mathrm{W}$ & 3.03 & 3.11 \\
\hline $\mathrm{Al}$ & 7.65 & 3.40 \\
\hline $\mathrm{Ti}$ & 10.86 & 3.69 \\
\hline $\mathrm{Nb}$ & 2.20 & 0.72 \\
\hline $\mathrm{Ta}$ & 3.66 & 2.47 \\
\hline $\mathrm{Ni}$ & 37.38 & 49.06 \\
\hline
\end{tabular}

For the solidified material (such as A3 in the phase diagram), during the rapid reheating process, when the reheating temperature was higher than the eutectic temperature but lower than the solidus temperature, based on the phase diagram, the precipitated $\gamma^{\prime}$ phase would generally dissolve into the $\gamma$ matrix under slow cooling rate. And there would be no liquid during the reheating process. However, the $\gamma^{\prime}$ phase usually have no sufficient 
time to dissolve completely into the matrix. The actual solidus curve was the blue line hd in the phase diagram. As shown in Figure. 4(c), the remaining $\gamma^{\prime}$ phase near the grain boundaries would react with the $\gamma$ matrix to produce a continuous low-melting point eutectic liquid film along the grain boundary when the previous deposit was reheated to the eutectic reaction temperature. The low melting point eutectic film weakened the grain boundary cohesion and led to a decrease in the ductility of the LSF part. Therefore, when the thermal stress exceeded the critical tensile stress, the liquation film would be undrawn, causing cracks along the grain boundary. And this analysis correlated well with the characteristics of narrow cracks in Figure. 1.

\section{Conclusions}

The main conclusions of this study are as follows:

1) FGH 98 superalloy parts were fabricated by LSF. It was found that the main defect in the LSF FGH 98 part was cracks, and two kinds of cracks were found on the cross section.

2) The temperature and stress fields were simulated. The simulation result has demonstrated that the LSF part experienced a series of periodic temperature and stress cycles.

3) The crack mechanism during the LSF process was explained according to the phase diagram and simulation results. It can be concluded that the micro segregation caused by the rapid heating and cooling rates was the main reason for the crack defect.

\section{Acknowledgements}

This work was supported by the National Key R\&D Program of China (2018YFB1105900) and the National Natural Science Foundation of China (51575308).

\section{References}

1. R.C. Reed, Superalloys: fundamentals and applications (Cambridge University Press, Cambridge, 2006)

2. E.O. Ezugwu, Int. J. Mach. Tools Manuf. 45, 13531367 (2005)

3. D. Zhu, X. Zhang, H. Ding, Int. J. Mach. Tools Manuf. 64, 60-77 (2013).

4. W.D. Huang, X. Lin, J. Chen, Z.X. Liu, Y.M. Li, Laser solid forming technology (Northwestern Polytechnical University Press, Xi'an, 2007)

5. M. Alimardani, V. Fallah, M. Iravani-Tabrizipour, A. Khajepour, J Mater Process Technol. 212, 113-119 (2012)

6. R. Kainuma, M. Ichinose, I. Ohnuma, K. Ishida, Mater Sci Eng A. 312, 168-175 (2001)

7. K. Otsuka, X. Ren, Prog Mater Sci. 50, 511-678 (2005) 